\section{Ecología en acción. Una experiencia educativa}

\section{Ecology in action. An educational experience}

\author{
Antonio Martín Ezpeleta \\ Universidad de Valencia \\ España \\ anmarez@uv.es \\ https://orcid.org/0000-0003-0210-3399 \\ Yolanda Echegoyen Sanz \\ Universidad de Valencia \\ España \\ yoesanz@uv.es \\ https://orcid.org/0000-0002-3729-460X
}

Cómo citar este artículo:

MARTÍN EZPELETA, A. y ECHEGOYEN SANZ, Y. (2020). "Ecología en acción. Una experiencia educativa", Pangeas. Revista Interdisciplinar de Ecocrítica, 2, pp. 74-88.

https://doi.org/10.14198/pangeas2020.2.06

\section{RESUMEN}

Los autores, profesores de Literatura y Ciencias Naturales en la Facultad de Magisterio de la Universidad de Valencia, han llevado a cabo una experiencia docente basada en la Ecocrítica, cuyo diseño y resultados se presentan en este trabajo. Así, además de desarrollar el planteamiento teórico-metodológico interdisciplinar que fundamenta esta experiencia, se describen las actividades didácticas generadas a partir del cuento "Anaconda" (1921), de Horacio Quiroga, que se han implementado en este curso 2016-2017. Estas intentan potenciar una lectura que prima la voz de la naturaleza, que esta vez toma cuerpo en los diálogos y reflexiones de las serpientes. Se rompe así esa imagen atávica que une los ofidios con el mal, coadyuvando al mismo tiempo la empatía y la creación de un nuevo punto de vista que demuestra que el verdadero enemigo de la biodiversidad es el hombre. Valores ecológicos medulares en la Década de la Educación para el Desarrollo Sostenible de las Naciones Unidas (2005-2014), que ya dejó bien a las claras la necesidad de primar la protección del medio ambiente y la preservación de la biodiversidad en todos los niveles educativos. Así, parece especialmente relevante colaborar en concienciar de todo ello a los maestros del futuro, que también pueden incorporar los presupuestos de la Ecocrítica en su bagaje formativo.

Palabras clave: ecocrítica; educación ambiental; educación literaria; educación científica; formación de profesores.

\begin{abstract}
The design and results of an ecocriticism-based teaching experience carried out by the authors, professors of Literature and Natural Sciences at the Faculty of Education of the University of Valencia, are presented. Thus, in addition to the interdisciplinary theoretical-methodological approach basing this experience, the didactic activities implemented in the academic year 2016-2017 and generated from the story "Anaconda" (1921) by Horacio Quiroga are
\end{abstract}


described. These try to focus on a reading in which the voice of nature prevails, in this case taking shape in the dialogues and reflections of the snakes. The atavistic image assimilating the ophidians with evil is broken and, at the same time, it is shown that the true enemy of biodiversity is mankind. These are core ecological values that were already revealed during the Decade of Education for Sustainable Development of the United Nations (20052014), where the need to prioritize the protection of the environment and the preservation of biodiversity at all educational levels was stated. Thus, it seems especially relevant to collaborate in raising awareness among the teachers of the future, who can also incorporate the Ecocriticm assumptions into their training background.

Keywords: ecocriticism; environmental education; literary education; scientific education; teacher training 


\section{Ecocrítica y educación. El proyecto "Ciencias y letras"}

Los Estudios culturales han impulsado un cambio de rumbo en las corrientes críticoliterarias al incorporar a los propedéuticos análisis un fin práctico y social. El proceso de ampliar el corpus de estudio añadiendo obras pertenecientes a otras esferas artísticas, como la del cine particularmente, y nuevos públicos receptores (ya no se convocan solo sectores cultos) se ha demostrado como un enriquecimiento crítico extraordinario, que ha cuajado en diversas disciplinas críticas de gran pujanza. Este pragmatismo es precisamente el que atraviesa la corriente de la Ecocrítica, que ni mucho menos muere en la constatación de los problemas medioambientales en productos culturales; sino que, antes bien, es un ejercicio de reflexión y concienciación. Así, podríamos afirmar que Estudios culturales y educación es un binomio indisoluble. Sin embargo, como Henry Giroux reclamaba hace más de veinte años en su importante artículo "Doing Cultural Studies. Youth and the Challenge of Pedagogy" (1994), el necesario diálogo que debe fluir entre las facultades de Educación y el mundo de los Estudios culturales no ha terminado de intensificarse. En el ámbito anglosajón, y centrado en la aportación de la Ecocrítica, todavía está lejos de ser satisfactorio, como desarrolla con un espíritu muy crítico Orr (2004 y 2010) o, entre otros, Goleman, Benett y Barlow (2012), Saylan y Blumstein (2011) o Scott (2010).

El caso en España no es muy distinto. Sí se observa cómo este tipo de estudios ecocríticos empiezan a emerger; no solo en los departamentos de Teoría de la literatura o Literatura Comparada, también en los departamentos de Filología Inglesa, aunque muy poco aún en los de Lenguas Romances. Y lo cierto es que el decidido impulso de proyectos como la Asociación Internacional Iberoamericana de Literatura y Ecocrítica, la revista Ecozon@ o el grupo de investigación GIECO liderado por Carmen Flys Junquera de la Universidad de Alcalá (al que debemos publicaciones básicas; véase ahora el estado de la cuestión en Flys Junquera, 2010, completado por Prádanos, 2016, entre otros trabajos de gran interés del mismo escritos al otro lado del Atlántico: 2012, 2015...) han cimentado la especialidad de la Ecocrítica en España. Pero este movimiento tampoco ha terminado de llegar a las facultades de Educación.

Esto no deja de ser curioso, pues la protección del medio ambiente ha pasado a ser una competencia transversal en muchos planes de estudio de Magisterio. Y es que sí se constata una apuesta por incorporar estos asuntos de protección del planeta en los currículos oficiales (Martínez Agut, 2007; UII et al., 2009, 2010); pero solo desde el punto de vista de las Ciencias, donde se cuenta con numerosas e interesantes aportaciones (Vilches y Gil Pérez, 2010, 2015...). Con todo, la conclusión que arroja el estudio de Ull et al. (2014), centrado en el caso de la Universidad de Valencia, es realmente sintomática al constatar que gran parte del profesorado siente como ajeno el problema de la sostenibilidad y que, incluso habiendo interés por parte de algunos docentes, estos no saben cómo integrarlo en sus clases.

Para el caso de las Letras, hay que reivindicar esa fructífera relación que se ha establecido históricamente entre la Teoría de la literatura y la Didáctica de la literatura, como han puesto de manifiesto numerosos autores (Mendoza, 2004; Martín Vegas, 2009; Ballester, 2015...). No obstante, falta dar ese paso que reclamamos: la asimilación de las aportaciones de la Ecocrítica y su transposición didáctica, según la terminología de Yves Chevallard (1985), para impulsar su aprovechamiento educativo. Contamos, eso sí, con algunos trabajos que han comenzado a explorar estas posibilidades, como los de Martos y Martos (2016) 
o Martos, Campos y Ezquerro (2017), además de los acerca-mientos al corpus de literatura infantil y juvenil de temática ecológica que han preparado Laso y León (2010) o Alves Bedendi (2011).

En este contexto es donde se incardina el proyecto "Ciencias y Letras", que hemos impulsado en la Facultad de Magisterio de la Universidad de Valencia ${ }^{1}$. El proyecto, que cuenta con nueve profesores de diferentes disciplinas (tres de Lengua y literatura; otros tres de Ciencias Sociales; y tres más de Ciencias Experimentales), se ha propuesto diseñar materiales didácticos inspirados en la integración de las Ciencias y las Letras, así como explorar sus posibilidades educativas en las aulas de Magisterio. $Y$ para este fin resulta fundamental la asimilación, como queda explicado, de los estudios de Ecocrítica, así como la apuesta por planteamientos inter/trans-disciplinares.

Desde el punto de vista pedagógico, la metodología que se impone es el denominado Aprendizaje Basado en Fenómenos (phenomenon-based teaching), que supone integrar en torno a fenómenos mundiales conocimientos y competencias de muy diversas disciplinas. Así, los fenómenos del cambio climático o la protección de la biodiversidad no solo se estudian a partir de las disciplinas de la Biología, la Física, la Economía o la Ecología; sino también desde la Historia, la Literatura o el Cine, por ejemplo (sobre este planteamiento metodológico, véase "Horizonte 2020, un nuevo modelo pedagógico" y Marina, 2016). En suma, el proyecto "Ciencias y Letras" está intentado desarrollar una nueva manera de enseñar, que acompaña de la experimentación de la misma con el fin de valorar su rendimiento $y$ aprovechamiento. Pero pasemos ya a contextualizar nuestra propuesta educativa.

\footnotetext{
${ }^{1}$ https://yoesanz.wixsite.com/cienciasyletras-uv
}

\section{Propuestas didácticas ecocríticas: “Anaconda” de Horacio Quiroga}

\subsection{Contextualización}

La clave de estas actividades didácticas que aquí presentamos radica en el hecho de que se han implementado a la vez en asignaturas prototípicas de las Ciencias y las Letras en la Facultad de Magisterio, como son "Ciencias Naturales para maestros" y "Formación literaria para maestros". Se trata de asignaturas de las denominadas "básicas", que se imparten en los primeros cursos de los grados de Maestro en Educación Infantil y Educación Primaria (en el caso del plan de estudios de la Universidad de Valencia, en ambos casos en segundo). La idea es, pues, vencer la organización curricular a partir de asignaturas, departamentos o áreas de especialización, y demostrar que los textos literarios son un recurso de primer orden para trabajar las Ciencias y que los contenidos científicos y la reflexión sobre la protección del medio ambiente no son incompatibles ni mucho menos con las clases de literatura. $Y$ es que los presupuestos de la Pedagogía crítica ya pusieron de relieve el hecho de que renovar los procesos de enseñanza y aprendizaje exigía también cambiar el sistema educativo. Esta denominada pedagogía liberadora (autores como Paulo Freire, 1989; o el citado Henry Giroux, 2005) en el fondo es incompatible con un sistema curricular inflexible, estanco y basado en el estudio monográfico y no en el conocimiento horizontal e interdisciplinar. En cualquier caso, el objetivo último, como no puede ser de otra manera, es impulsar el conocimiento de los alumnos en materias tan relevantes como Lengua, Literatura, Ciencias; pero siempre entremezclando saberes y supeditándolos a una formación holística, real y ética, que incluye aspectos relacionados con la Ecología y protección del medio ambiente, pero también con el civismo o la empatía. 
Tal y como hemos apuntado previamente (Martín Ezpeleta y Echegoyen Sanz, en prensa), conviene, antes de nada, señalar que la naturaleza de la facultad de Magisterio invita especialmente a que sus alumnos, los maestros del futuro, interioricen las bondades de una manera de aprender que está completamente alineada con la realidad de los maestros en los colegios. Y es que, más allá de los planes de estudio de estas facultades, la tarea de los profesionales de la educación en las escuelas es integrar contenidos y competencias muy heterogéneos en sus clases, que no están tan férreamente compartimentadas en asignaturas y especialidades como sucede en la Educación Secundaria o Superior.

Es decir, esa mencionada metodología basada en fenómenos está perfectamente alineada con el quehacer profesional de unos futuros maestros que muchas veces se quejan de no saber para qué sirven los conocimientos técnicos en materias especializadas que cursan en los grados de Maestro en Educación Infantil y Educación Primaria, que reúnen prácticamente todas las ramas del saber: Lengua, Literatura, Química, Biología, Música, Psicología... El aprendizaje basado en fenómenos permite, pues, romper los muros departamentales y supeditar todos estos conocimientos técnicos en proyectos que ayuden a los alumnos de Magisterio a contestar a esa pregunta y, según queda dicho, a interiorizar una nueva metodología susceptible de ser aplicada en su quehacer profesional inmediato.

Pues bien, el proyecto ha sido llevado a cabo durante el curso lectivo 2016-2017 con cien alumnos de la Facultad de Magisterio de la Universidad de Valencia, repartidos en las asignaturas de "Formación literaria para maestros", impartida por Antonio Martín Ezpeleta, que es una asignatura obligatoria de 6 créditos del Grado de Maestro en Educación Primaria, y "Ciencias Naturales para maestros", también obligatoria del
Grado de Maestro en las especialidades de Infantil y Primaria, de 9 créditos e impartida por Yolanda Echegoyen Sanz. En este contexto de Literatura y Ciencia aplicadas es precisamente donde, a nuestro juicio, ha de incardinarse el mundo de la Ecocrítica en el ámbito de Magisterio. Su naturaleza híbrida científico-literaria, pero sobre todo su vocación social y de protección del planeta, supone que la lectura y comprensión de textos literarios atravesados por conocimientos técnicos y éticos relacionados con la Ecología y la sostenibilidad es un inmejorable punto de partida para sensibilizar a unos jóvenes que están encargados de transmitir este mensaje a miles de niños en su futuro profesional. El asunto ha sido explorado en la Literatura Infantil y Juvenil, donde contamos con libros tan exitosos como los de Jen Green y Mike Gordon (¿Por qué debo ahorrar energía?, ¿Por qué debo proteger la Naturaleza?, ¿Por qué debo reciclar? o ¿Por qué debo ahorrar agua?); pero el paso previo a utilizar estos materiales para niños es concienciar a los maestros, y para ello puede ser muy útil utilizar textos como el cuento de "Anaconda", de Horario Quiroga, según inmediatamente explicaremos.

\subsection{Desarrollo}

En ambas asignaturas se partió de la lectura del cuento "Anaconda" para desarrollar diferentes actividades complementarias. En el caso de "Formación literaria para maestros", la unidad didáctica comenzó con la lectura tan solo de los primeros párrafos del cuento. Estos sirven de presentación de la trama, pero no incluyen a la protagonista, el personaje epónimo de Anaconda, sino a Lanceolada, que es una víbora. Pocos alumnos se percataron de este hecho, y mostraron desconocer que las serpientes se dividen en víboras y culebras (en cuya familia está la anaconda), que, 
como el cuento ilustra magistralmente, son dos especies muy parecidas, aunque con notables diferencias.

En este sentido, muchos alumnos aprendieron en clase de "Formación literaria para maestros" que las víboras inyectan veneno para defenderse, mientras que las culebras atacan por asfixia. También se percataron de que estas últimas son mucho mayores en tamaño e inmunes al veneno de las víboras, por lo que resultarían victoriosas en una hipotética pelea entre estas familias de ofidios. Respecto a su relación con los humanos, también se concluyó que la víbora es mucho más peligrosa $\mathrm{y}$, salvo en las creaciones de Hollywood, las culebras están más relacionadas con animales de compañía, por mucho que las mayores puedan ser peligrosas para niños, por ejemplo. En fin, toda esta información, que salió a colación antes de realizar la actividad, fue rápidamente conectada por los alumnos con las populares aventuras del personaje televisivo Frank de la Jungla, que no deja de ser un divulgador científico.

Podría afirmarse, pues, que hubo un paso previo de asimilación de información biológica que sirvió a los alumnos para desarrollar la actividad principal de la unidad didáctica, que no era otra que la redacción de su particular cuento de "Anaconda" (el cuento de Quiroga solo se leyó al final, cuando sirvió para contrastar los prejuicios y creatividad de cada alumno), que había de ir enmarcado con estos primeros párrafos originales que el profesor les facilitó:

Eran las diez de la noche y hacía un calor sofocante. El tiempo cargado pesaba sobre la selva, sin un soplo de viento. El cielo de carbón se entreabría de vez en cuando en sordos relámpagos de un extremo a otro del horizonte; pero el chubasco silbante del sur estaba aún lejos. Por un sendero de vacas en pleno espartillo blanco, avanzaba Lanceolada, con la lentitud genérica de las víboras. Era una hermosísima yarará de un metro cincuenta, con los negros ángulos de su flanco bien cortados en sierra, escama por escama. Avanzaba tanteando la seguridad del terreno con la lengua, que en los ofidios reemplaza perfectamente a los dedos (Quiroga, 1991: 185).

Al día siguiente, la primera preocupación de Lanceolada fue el peligro que con la llegada del Hombre se cernía sobre la Familia entera. Hombre y Devastación son sinónimos desde tiempo inmemorial en el Pueblo entero de los Animales. Para las víboras en particular, el desastre se personificaba en dos horrores: el machete escudriñando, revolviendo el vientre mismo de la selva, y el fuego aniquilando el bosque en seguida, y con él los recónditos cubiles.

Tornábase, pues, urgente prevenir aquello. Lanceolada esperó la nueva noche para ponerse en campaña. Sin gran trabajo halló a dos compañeras, que lanzaron la voz de alarma. Ella, por su parte, recorrió hasta las doce los lugares más indicados para un feliz encuentro, con suerte tal que a las dos de la mañana el Congreso se hallaba, si no en pleno, por lo menos con mayoría de especies para decidir qué se haría (1991: 187).

A partir de aquí, el texto principal desaparecía, hasta estas últimas líneas finales, prototípicas de las sagas de aventuras: "Pero la historia de este viaje remontando por largos meses el Paraná hasta más allá del Guayra, más allá todavía del golfo letal donde el Paraná toma el nombre de río Muerto, la vida extraña que llevó Anaconda y el segundo viaje que emprendió por fin con sus hermanos sobre las aguas sucias de una gran inundación, toda esta historia de rebelión y asalto de camalotes, pertenece a otro relato" (1991: 220).

En este punto, pues, se desarrolló un pequeño taller de escritura (sobre estos, 
véase Rodari, 1985; Sánchez-Enciso, 1988; Delmiro, 2002; o Lardone y Andruetto, 2007; así como los estudios de Owens, 2001; Dyment y O'Connell, 2007; Newcomb, 2012; o Savageau, 2013, estos últimos centrados en la educación ambiental), que pasaba por secuenciar la tarea de redactar el cuento, invitando a los alumnos a utilizar y asimilar los tecnicismos narratológicos que se habían estudiado en la asignatura. Así, se partió de la diferenciación entre trama y argumento, facilitando que tomaran decisiones sobre los componentes de la narración (el narrador, los personajes principales y secundarios, el espacio, el tiempo) y el argumento (el orden, la secuenciación del tiempo, el discurso de los personajes, etcétera). Es decir, no podían partir de la hoja en blanco, sino de una serie de respuestas a las preguntas previas sobre la trama, que debían condensar también en un resumen del cuento que iban a escribir.

Lo cierto es que la mayor parte de los textos generados por los alumnos muestra cómo el germen de la historia en el principio que leyeron, a despecho de que no había aparecido Anaconda todavía, sí lo captaron, y que se condensa en el fragmento citado: "Al día siguiente, la primera preocupación de Lanceolada fue el peligro que con la llegada del Hombre se cernía sobre la Familia entera. Hombre y Devastación son sinónimos desde tiempo inmemorial en el Pueblo entero de los Animales" (1991: 187). Este es un ejemplo:

Tema: Los celos son el enemigo.

Personajes: principales: Anaconda y Lanceo-lada; secundarios: el hombre y el bebé.

Espacio-tiempo: se sitúa en la selva, cerca de la casa.

Resumen:

Anaconda, a pesar de ser una más entre todas las serpientes, siempre se creía superior al resto e intentaba ser la líder, por lo que Lanceolada no quiso avisarle del peligro. Entre todas las serpientes convocadas para el supuesto ataque contra el hombre se encontraba Mamba, la mano derecha de Anaconda. Mamba le informa a Anaconda de lo que va a pasar y le cuenta que Lanceolada no ha querido que se enterase. Anaconda sin pensárselo dos veces va directa a atacar a Lanceolada. Se enfrentan en una gran pelea justo debajo de la habitación del bebé. Con los fuertes ruidos, este se despierta y comienza a llorar. El hombre, padre de la criatura, al ir a ver qué le pasa descubre a las serpientes y las mata (M.M.).

Durante la elección de los componentes de la trama, el profesor colaboró con los alumnos, insistiendo en los conceptos narratológicos y en cómo cada decisión repercutía, obligando muchas veces a reestructurar el argumento. Del mismo modo, también acompañó la redacción de una parte del cuento, que preferiblemente no tenía que ser la que enlazaba inmediatamente con el texto original; sino un momento clave, el clímax, de su argumento, que es el que tenían que esforzarse en redactar con mayor peso de la connotación. Traemos a colación un ejemplo, redactado por el mismo alumno (M.M.) y pensando en un público receptor infantil. En este caso el alumno ha decidido preparar un texto en la modalidad más mimética, el estilo directo, que elige para simular la tensión de una especie de guerra entre los hombres y las serpientes, que se expresan en un atinado tono entrecortado y nervioso; nótese también cómo el alumno parece recordar la lección de los nombres propios denotativos...:

- ¿Criptonita, Criptonita! -sonaba en el walkie-talkie de Criptonita-. Tenemos un problema. Es Juan Carlos Escopeta. Tenemos dos heridos.

-Sí, Lanceolada, te recibo. Enseguida mando un aviso a la central: "Tenemos dos heridos. Necesitamos dos serpiambulancias y cuatro patrullas de ataque si no 
queremos quedarnos sin más compañeros y sin provisiones de alimentos".

Y Criptonita se fue alejando dando órdenes.

-Pero... ¿qué opina la Gran Anaconda? -dijo Lanceolada-. Ya sabes que sin su opinión no podemos dar ningún tipo de órdenes al ejército.

En fin, la actividad concluyó con la lectura de algunos textos, que sirvió para ver las posibilidades creativas que ofrecía ese principio, que, venciendo el relativismo, se orientaba en torno al eje del respeto del medio ambiente, la preservación de la biodiversidad y el papel dañino que muchas veces desempeña el hombre en las selvas vírgenes. La actividad concluyó, según queda advertido, con la lectura del cuento original, a la que siguió la explicación de algunas notas sobre la obra de Horacio Quiroga y su extraordinaria importancia en la Historia del cuento universal, así como la reflexión sobre esta figura que une la tradición con la modernidad. En este punto, se trazó también una pequeña constelación literaria (Jover, 2007 y coord. 2009), que unió las fábulas tradicionales con la temática de las denominadas novelas de la tierra (la más famosa es La vorágine, de José Eustasio Rivera). Y también hubo lugar para sintetizar la evolución del género del cuento moderno de la mano de Edgar Allan Poe y la cadena que en él comienza y que une al propio Horacio Quiroga y otros autores del boom hispanoamericano como Jorge Luis Borges, Julio Cortázar o Gabriel García Márquez, además del novelista Alejo Carpentier y su inmarcesible Los pasos perdidos, que tanto tiene que ver con la temática y autores mencionados. En suma, una breve lección de Historia de la literatura universal, perfectamente atravesada por conceptos teórico-literarios, que ayudó a los alumnos a conectar con el tema sobre la narrativa estudiado, además de con otras lecturas realizadas durante el curso de varios de los autores mencionados.

El resultado, que más adelante se ponderará, fue positivo. Los alumnos, que gustan de estas clases prácticas y de darle la vuelta al tapiz de la escritura para ver cómo está tejido, supieron utilizar los conceptos, desarrollar la creatividad y entroncar estos aprendizajes con las notas más eruditas que ofreció el profesor, y que parece que supusieron lo que en el argot educativo se denomina un aprendizaje significativo, es decir, un aprendizaje que parte de cosas sabidas y vividas. Por último, se consiguió, mediante estas ficciones narrativas, empatizar con las serpientes, combatiendo esa imagen atávica que las relaciona con el mal, y que ha sepultado su necesaria función en la cadena atrófica e incluso sus beneficios directos para el mundo de la Medicina, que, como los alumnos descubrieron en clase, explican que su imagen esté presente en las farmacias.

Por su parte, en "Ciencias Naturales para maestros" los alumnos leyeron el cuento antes de comenzar la actividad. Esta lectura resultaba muy pertinente para desarrollar asuntos del núcleo temático de la biodiversidad, que es el que se estaba estudiando. En concreto, se aprovechó para ilustrar aspectos relacionados con el segundo nivel de la biodiversidad, el intraespecífico, por la multitud de especies de serpientes que aparecen en el texto. La relación de estas con el hombre, al que se alude constantemente como "la muerte" en el texto, servía perfectamente al cometido de ejemplificar la pérdida de biodiversidad por la acción directa del ser humano, uno de los principales problemas ambientales junto con el calentamiento global, la sobreexplotación de los recursos naturales o la generación de residuos, todos ellos aspectos clave en este tema de "Sostenibilidad y medio ambiente". 
La actividad, al igual que en el caso de "Formación literaria para maestros", se basó en la creatividad y los talleres literarios. En este caso, se trabajó por parejas la redacción de un cuento destinado a niños que siguiera las instrucciones de que el protagonista fuera un animal y que el tema tratase de concienciar sobre algún problema medioambiental de los estudiados en clase, que debía quedar suficientemente claro en una moraleja. Se invitó a los alumnos a elegir el animal protagonista, así como los personajes secundarios, de los que debían describir sus principales características físicas y psicológicas (una descripción clásica prosopográfica y etopéyica).

Así, se escribieron cuentos sobre distintos tipos de animales terrestres y marinos. Anecdóticamente los animales más elegidos para el papel de héroes fueron la tortuga y la abeja. Generalmente las tramas incluían a un protagonista que era víctima de la acción del hombre, aunque en un par de ocasiones también se registra un animal antagonista, que contamina su propio entorno. Por lo demás, en la mayoría de las historias aparecían seres humanos, que adoptaban dos roles distintos: por un lado, aquellos que eran los causantes del problema que sufrían Ios animales; por otro, seres humanos, en muchas ocasiones niños, que ayudaban a los animales y los protegían de la acción negativa de algunos adultos. Es curioso notar también que la gran mayoría de las historias se desarrollaban en el tiempo actual (solo un par de ellas se sitúan en el futuro, con un deje apocalíptico) y que tenían lugar en prácticamente todos los continentes (en cuatro ocasiones, se opta por situar la historia en Valencia y sus alrededores). En fin, quizá por la influencia de la lectura del cuento "Anaconda", también llama la atención la coincidencia en describir asambleas de distintos animales que se reúnen para encontrar la solución al problema que les acucia.
En relación a los temas tratados, los que con más frecuencia aparecieron fueron la contaminación y los residuos, la deforestación y la modificación de los ecosistemas, aunque también hubo cuentos que versaron sobre la pérdida de biodiversidad, el calentamiento global, la violencia contra animales, la sobreexplotación de recursos o el uso de pesticidas. Reproducimos, a modo de ejemplo, dos de las historias creadas, que, a despecho de no terminar de redactar correctamente las moralejas (no se explicó en clase nada sobre este particular, que se dio por sabido), muestran cómo asimilan información científica, literaria y didáctica en una actividad, por lo demás, que resultó estimulante a los alumnos:

"En busca de los bebés perdidos"

Era un día soleado de verano en las costas de Turquía. Alli vivía Manuelita, una tortuga que dentro de poco iba a cumplir su mayor sueño, ser mamá.

Cada día que pasaba se iba acercando el momento de recibir a las nuevas tortugas, hasta que llegó el día [en] que los bebés llegaron, en la playa de Iztuzu. Manuelita decidió dejar sus cuatro huevos muy protegidos, y con mucha pena los dejó allí para volver dentro de dos meses, para ver si los pequeños ya habían salido del huevo.

Pasado el tiempo, Manuelita volvió, y se encontró una playa muy distinta a la que recordaba. Estaba llena de basura y muy descuidada, prácticamente no podía ver el sitio donde dejó los huevos. Entonces, le entró mucho miedo. Allí conoció a la gaviota Carlota que le contó que durante esos meses muchas personas habían ido a la playa y habían tirado basura, pero que, por suerte, sus bebés estaban a salvo gracias a la ayuda de personas que cuidaban el medio ambiente, las cuales habían movido los huevos a la playa de Dalyan.

En ese momento Manuelita era capaz de cualquier cosa por encontrarse con sus hijos y, pese a lo alejada que estaba la playa de Dalyan, fue en busca de los bebés. Allí los 
encontró sanos y salvos, y finalmente decidieron quedarse a vivir en esa playa, que no estaba contaminada.

Moraleja: Debemos enseñar a los alumnos a cuidar nuestras playas y respetar el hábitat de los animales que viven allí (A.J. y S.F.).

\section{“La osa Rosa”}

Rosa es una pequeña osa polar que vive en Canadá, en los glaciares. Un día, durante la ceremonia del inicio a su edad adulta, se ve obligada a cazar una foca. No obstante, ella se niega a hacerlo y así conoce a la foca Agustina, que se convertirá en su mejor amiga.

Su nueva amiga, tras unos meses de amistad, le revela su mayor secreto: es científica y ha estado creando una máquina del tiempo. Entonces, como a Agustina le da un poco de miedo, le propone a Rosa viajar al futuro para ver lo bonito que estará el mundo.

Rosa acepta y al día siguiente inicia su aventura. Viaja al año 2017 y descubre que el mundo no es como lo habían imaginado. Su casa ha desaparecido, se ha derretido. Todos los osos han tenido que mudarse al norte y están muy apretados. Rosa decide ir a ver la casa de Agustina y ve que su familia está hablando de que tienen que mudarse. También oye que el hermano de Agustina ha muerto por el deshielo del glaciar donde estaba viviendo. Rosa está muy triste y durante su paseo se encuentra muchas bolsas de plástico por el suelo y latas en el agua. No entiende qué hacen todas esas cosas allí y la razón por la que todos sus amigos están menos felices, pero a los humanos les parece dar igual.

Vuelve con Agustina y deciden trazar un plan juntas. Escriben un breve libro en el que cuentan su vida y el contraste de cómo cambiará dentro de 30 años si nadie hace nada para evitarlo. La dejan en el escritorio de un barco de exploración científica.

Moraleja: Debemos evitar tirar basura al mar y concienciarnos de que todas nuestras acciones tienen repercusiones, a pesar de que pasen a kilómetros de distancia (L.E. y M.C.).
Para concluir la presentación de estas actividades, contamos también con la opinión de los propios alumnos, que sucintamente analizamos en el siguiente epígrafe.

\subsection{Valoración}

Con el objeto de monitorizar la experiencia, se pasaron dos cuestionarios a los alumnos, uno antes y otro después de las actividades didácticas, con algunas preguntas comunes en las dos asignaturas.

\subsubsection{Cuestionario previo}

En el primer cuestionario, se fue acercando el asunto de la separación y posible integración de las Ciencias y Letras a partir de preguntas de respuesta cerrada, como, por ejemplo, si habían encontrado sencilla la elección en la Enseñanza Secundaria de optar por Ciencias o Letras para continuar su formación. Entre otras respuestas, sorprende que la amplia mayoría de los alumnos, un $76 \%$, considere que no es adecuada la tradicional separación entre Ciencias y Letras, que argumentan en ocasiones con atinadas explicaciones como estas: "Deberían estudiarse de una manera más integrada. Podemos emplear no todo, pero algunos aspectos científicos para las Letras y viceversa. En la vida todo aparece junto y en interacción, tanto Letras como Ciencias" (S.D.); 0 “Ambas disciplinas (aunque algunos no lo quieran ver) están estrechamente ligadas y se necesitan una a la otra para conseguir lo que realmente importa: aprender, comprender, crear, descubrir..." (S.R.).

Por lo demás, para observar los prejuicios existentes, se les pidió que ordenasen según su preferencia una serie de animales, a saber, el cerdo, la serpiente, el conejo, el perro y la araña. La inmensa mayoría, casi el $90 \%$, indica que entre esos el perro es su animal favorito (el conejo y el cerdo ocupan el 
segundo y tercer puesto). No sorprende que la mayor parte de los alumnos asocie la serpiente con veneno y peligro, en un $49 \%$ y $41 \%$ respectivamente; aunque un $7 \%$ lo relacione con una medicina y en un $3 \%$ con virtudes como la astucia o la inteligencia.

Resulta curioso, eso sí, que en general los alumnos no conocen historias donde las serpientes aparezcan como protagonistas. Además, la mayoría, un $71 \%$, anticipa que en el cuento de "Anaconda" estos animales resultarán peligrosos y dañinos, con argumentos como los que siguen: "En todas las historias la serpiente siempre hace de malvada porque se considera un animal peligroso" (P.D.); "Normalmente la serpiente suele actuar como una enemiga del hombre, por lo tanto en muchos casos se le atribuye un papel antagonista. También en la Biblia aparece el pasaje donde una serpiente hizo que Adán y Eva mordieran la manzana y abandonaran el paraíso" (E.A.). Los que contestan que aparecerá como un animal simpático es, precisamente, por contraposición: "Creo que actuará de manera diferente para cambiar nuestra visión sobre las serpientes ya que tenemos una visión negativa hacia ellas" (S.R.); "Pienso que este cuento pretenderá hacernos ver que la serpiente no es un animal malo, que también tiene sentimientos y que es igual de válido que cualquier otro" (K. S.).

A los alumnos de "Ciencias Naturales para maestros" se les pidió que nombrasen historias (libros, series o películas) en las que apareciesen animales como protagonistas. Destaca que las más conocidas son películas de Disney con formato de dibujos animados, bien clásicas como "Bambi", "101 Dálmatas" o "Dumbo", o más modernas como "Madagascar", "Ice Age", "Buscando a Nemo", “EI libro de la selva" o "El rey león”, estas dos últimas las más citadas con diferencia. Aparecen también algunas películas o series con personajes reales como "Beethoven", "Liberando a Willy" o "Rex, un policía dife- rente". En un par de ocasiones se nombran las fábulas, en concreto la de la liebre y la tortuga, y un alumno (E.A.) incluso trae a colación el nombre de Samaniego. En cuanto a la pregunta de por qué creen que se utilizan animales como personajes, la respuesta mayoritaria es porque llaman la atención de los niños al ser personajes "cercanos", "simpáticos" y "que inspiran ternura". Reproducimos aquí algunas opiniones: "Creo que el hecho de utilizar animales como personajes es para que las obras sean más creativas e imaginativas y los niños conecten mejor con estas sin que nadie se sienta identificado directamente y así poder transmitir lecciones morales, valores, educación..." (S.R.); "Creo que ponen animales para que asociemos 0 nos inculquen qué papel en la sociedad tiene cada animal. Por ejemplo, un burro siempre es el tonto, la tortuga es lenta, etc." (N.M.)"; "Porque en muchas ocasiones es una forma diferente y que llama la atención para transmitir variedad de sentimientos y valores, y creo que los humanos empatizamos con ellos de forma positiva si son buenos y negativa si son peligrosos" (A.L.).

\subsubsection{Cuestionario final}

El segundo cuestionario retomaba el asunto de las Ciencias y Letras, pero una vez que los alumnos habían llevado a cabo en el aula las actividades descritas. Los datos que siguen son claves: casi la totalidad del alumnado, un 93\%, considera que la Literatura puede ayudar a aprender Ciencias; y el $7 \%$ restante no tiene una opinión formada. El texto de "Anaconda" gustó "mucho" o "bastante" al 64\% de los discentes. En cuanto a la integración de las Ciencias y las Letras en clase, el $41^{\prime} 7 \%$ de los alumnos de "Formación literaria para maestros" y el 62'5\% de "Ciencias Naturales para maestros" la consideran "muy importante"; y el $55^{\prime} 6 \%$ y el 29 ' $2 \%$ 
respectivamente, "bastante importante". Las razones de nuevo son muy indicativas de que el tema ha de estar presente en los planes de estudio de Magisterio: "Porque ambas son importantes para nuestra vida, por ello me parece una idea fantástica leer un libro en el que hable de Ciencias, porque al mismo tiempo que estás leyendo, estás ampliando tus conocimientos sobre algo que igual no conocías pero es interesante" (M. B.); "Creo en el aprendizaje significativo $\mathrm{y}$, por tanto, considero que el desarrollo cognitivo se consolida más fácil, adecuada y sólidamente relacionando las diferentes áreas de conocimiento, atendiendo a la multidimensionalidad del aprendizaje" (V.A.). Poco que añadir.

En fin, después de realizar la actividad, en la clase de "Ciencias Naturales para maestros" el 22'4\% y el 69'4\% afirma estar "muy" y "bastante" sensibilizado con la sostenibilidad y el cuidado del medio ambiente. En la clase de "Formación literaria para maestros", los porcentajes varían ligeramente: $38 \%$ y $57 \%$ respectivamente. Algunas de las estrategias que proponen los alumnos utilizar para fomentar el cuidado del planeta con sus futuros discentes son: "En primer lugar, creo que es fundamental que ellos vean cuál es mi actitud frente al medio ambiente, ya que tienden a tener actitudes de imitación, e imitarían mis comportamientos. Además, lo trataría como un tema transversal dentro del aula, estando siempre presente y otorgándole la importancia que se merece" (L.L.); o "Reciclar en clase, que cada semana aportaran ideas nuevas para reciclar los envases, hacer ver a los futuros alumnos lo que ocasionan poco a poco si no cuidan el planeta y mostrarles de forma visual las consecuencias y hacer un pequeño debate para que ellos sacaran sus propias conclusiones y soluciones que estén en sus manos" (A.J.).

De nuevo, más del 90\% afirma que utilizaría textos literarios en clase para conseguir ese propósito, acompañados de argumentos como, por ejemplo: "Sería un buen recurso. Las actividades de la vida diaria requieren tanto de habilidades lingüísticas como de razonamiento por lo que no tiene demasiado sentido trabajarlas como áreas aisladas. Además, aquellos alumnos a los que no les gusten demasiado las Ciencias o les resulten complicadas, les puede ayudar tratarlas como si fuesen una historia" (I.P.); o "Sí, por medio de cuentos los niños aprenden muchos conceptos que les hacen reflexionar y pensar. A través del cuento se despierta mucho su interés, les gusta mucho oírlos y se concentran en él, es un gran medio que utilizar y explotar en la Educación Infantil que nos beneficia a la hora de tratar numerosos ámbitos y conceptos" (L.Q.).

De hecho, un $46 \%$ de los alumnos de la clase de "Ciencias Naturales para maestros" incluye la actividad de escribir un cuento para niños con un animal como protagonista entre las tres que más les gustaron de las realizadas durante todo el curso: "La actividad que más me gustó fue la de elaborar un libro porqué me pareció gracioso ver aquello que mis compañeros habían elaborado y cómo en nuestra aula cuando seamos futuros/as docentes poder enseñar a nuestros/as alumnos/as de forma indirecta a cuidar del medio ambiente o las posibles repercusiones que puede suponer un mal uso de los recursos" (M.O.); "Me gustó mucho la del cuento infantil porque era una forma de trabajar el medio ambiente y la sostenibilidad en la escuela como futuros maestros que somos" (P.D.).

Parece claro que la actividad les ha estimulado y que encuentran interesante seguir profundizando en los temas de la sostenibilidad y el medio ambiente, así como en no desvincular las Ciencias y las Letras, que se ayudan mutuamente. Los docentes no podemos estar más de acuerdo. Pero recapitulemos para terminar nuestras conclusiones en un apartado a tal efecto. 


\section{Conclusiones}

Parece claro que Ecocrítica y educación son dos conceptos que se necesitan mutuamente, de la misma manera que la literatura y la sociedad muestran un indefectible vínculo. Los Estudios culturales nos han permitido crecer en el conocimiento de este vínculo. $Y$ es precisamente este camino de apostar por la función socializadora de la literatura el que se indica desde el mundo de la Didáctica de la literatura que puede tener mayor rendimiento educativo en las aulas actuales, en tanto en cuanto permite conectar la realidad de los alumnos con los textos, lo real con lo ficticio, en un juego intelectual de innegables beneficios.

En este sentido, esta experiencia educativa, incardinada en un proyecto innovador de mayor recorrido, pretende impulsar que las facultades de Magisterio sean más permeables a estos nuevos rumbos de la crítica literaria, como la Ecocrítica, que si bien cada vez son más conocidos en el mundo de la Teoría de la literatura española, son prácticamente ignotos en los ámbitos educativos. Y esto no deja de ser curioso, pues, según queda explicado, la Pedagogía crítica y los modernos presupuestos psicopedagógicos y didácticos no pueden estar más alineados con esta manera de explicar la literatura focalizando en su vertiente ética y social.
Por lo demás, el hecho de que en las facultades de Magisterio contemos con los maestros del futuro significa también una responsabilidad: la de aprovechar los espacios educativos que genera la lectura de textos relacionados con la naturaleza, | 86 especialmente con el cuidado del medio ambiente. Un camino que la Década de la Educación para el Desarrollo Sostenible de las Naciones Unidas inició, y que no es más que el principio de una nueva era que verá cambios educativos y el desarrollo de una tan necesaria conciencia sobre la sostenibilidad. En nuestras manos está preparar los materiales educativos pertinentes e implementar las prácticas docentes que la harán posible. 


\section{BIBLIOGRAFÍA}

ALVES BEDENDI, M. L. (2011). “Literatura infantil y educación ambiental. Contribución en la construcción de la identidad del ser humano". En Eventos pedagógicos, 2 (3), 59-69.

BALLESTER, J. (1991). La formación lectora y literaria. Barcelona: Graó.

CHEVALLARD, Y. (1991). La transposición didáctica. Buenos Aires: Aique.

DELMIRO, B. (2002). La escritura creativa en las aulas. En torno a los talleres literarios. Barcelona: Graó.

DYMENT, J., O'CONNELL, T. (2007). “Journal Writing on Wilderness Expeditions as a Tool for Sustainability EducationReflections on the Potential and the Reality". En Applied Environmental Education and Communication, 6 (2), 139-148.

FLYS JUNQUERA, C., MARRERO HENRÍQUEZ, J.M., BARELLA VIGAL, J. (eds.) (2010). Ecocríticas. Literatura y medio ambiente. Madrid: Iberoamericana / Vervuert.

FREIRE, P. (1989). La educación como práctica de la libertad, trad. L. Ronzoni. Madrid: Siglo XXI.

GIROUX, H. (1994). "Doing Cultural Studies. Youth and the Challenge of Pedagogy". En Harvard Educational Review, 64 (3), 278309.

(2005). Estudios culturales, pedagogía crítica y democracia radical, epílogo D. Kellner. Madrid: Popular.

Golema, D., Bennet, L., Barlow, Z. (2012). Ecoliterate. How Educator Are Cultivating Emotional, Social and Ecological Intelligence. San Francisco: Jossey-Bass.

"Horizonte 2020, un nuevo modelo pedagógico". Consultado en línea (28/10/2017):

http://www.educacionjesuitas.es/noticias /248-horizonte-2020-un-nuevo-modelopedagogico

JOVER, G. (2007). Un mundo por leer. Educación, adolescentes y literatura. Barcelona: Octaedro. (coord., 2009). Constelaciones literarias. Sentirse raro. Miradas sobre la adolescencia. Sevilla: Junta de Andalucía.

LARDONE, L., ANDRUETO M. T. (2007). La construcción del taller de escritura. Buenos Aires: Homo Sapiens.

LASO Y LEÓN, E. (2010). “La literatura infantil y juvenil: el nacimiento de una conciencia”. En Carmen Flys Junquera, José Manuel Marrero Henríquez y Julia Barella Vigal (eds.): Ecocríticas. Literatura y medio ambiente. Madrid: Iberoamericana / Vervuert, 339-370.

MARINA, J.A. (2016) “Humanidades. Una propuesta para volver a integrarlas en la educación". El Confidencial, 23 de febrero de 2016. Consultado en línea (28/10/2017):

http://www.joseantoniomarina.net/articul o/humanidades-una-propuesta-paravolver-a-integrarlas-en-la-educacion/

Martín Ezpeleta, A., Echegoyen Sanz, Y. “La educación científico-literaria. Ecocrítica y sostenibilidad en la novela Sayonara Sushi de Raül Romeva". En Letras hispanas, número monográfico sobre Ecocrítica, en prensa.

MARTÍN VEGAS, R. A. (2009). Manual de didáctica de la lengua y la literatura. Madrid: Síntesis.

MARTÍNEZ AGUT, M. P. et al. (2007). "Promoción de la sostenibilidad en los currícula de la enseñanza superior desde el punto de vista del profesorado: un modelo de formación por competencias". En Educatio siglo XXI. Revista de la Facultad de Educación, 25, 187-208.

MARTOS NÚÑEZ, E., MARTOS GARCÍA, A. (2016). "La perspectiva performativa en las ciencias sociales y en las prácticas alfabetizadoras de educación ambiental, literaria y patrimonial". En Caracteres. Estudios culturales y críticos de la esfera digital, 5 (1), 10-40.

MARTOS NÚÑEZ, E., CAMPOS FERNÁNDEZFIGARES, M., MARTÍNEZ EZQUERRO, A. (2017). "Superando la dicotomía entre humanidades y ciencias. De las 
narrativas míticas a la difusión científica a través de la cultura del agua". En CTS. Revista iberoamericana de ciencia, tecnología y sociedad, 12 (35), 177-182.

MENDOZA, A. (2004). La educación literaria. Bases para la formación de la competencia lectoliteraria. Málaga: Aljibe.

NEWCOMB, M. (2012). "Sustainability as a design principle for composition: Situational creativity as a habit of mind". En College Composition and Communication, 63 (4), 593-615.

"Phenomenal Education" (2017). Consultado en línea (28/10/2017): http://www.phenomenaleducation.info/p henomenon-based-learning.html

ORR, D. (2004). Earth in Mind. On Education, Environment and the Human Prospect. Victoria: Island.

. "¿Para qué sirve ahora la Educación Superior?". En La situación del mundo 2010. Cambio cultural: del consumismo hacia la sostenibilidad, Barcelona: Icaria, 155-167.

OWENS, D. (2001). Composition and Sustainability: Teaching for a Threatened Generation. Urbana: NCTE.

QUIROGA, H. (1991). Cuentos, ed. de Leonor Fleming. Madrid: Cátedra.

PRÁDANOS, L.I. (2012). “Decrecimiento o barbarie: ecocrítica y capitalismo global en la novela futurista española reciente". En Ecozon@. European Journal of Literature, Culture and Environment, 3 (2), 71-92.

. (2015a). "La enseñanza del español en la era del antropoceno: hacia la integración de la sostenibilidad en las clases de español como lengua extranjera". En Hispania, 98 (2), 333345.

. (2015b). "The Pedagogy of Degrowth. Teaching Hispanic Studies in the Age of Social Inequality and Ecological
Collapse". En Arizona Journal of Hispanic Cultural Studies, 19, 153-168.

. (2016). "Ecocrítica en los estudios literarios y culturales españoles contemporáneos. Una tendencia emergente desesperadamente necesaria". En La nueva literatura hispánica, 20, 269-288.

RODARI, G. (1985). Gramática de la fantasía. Introducción al arte de inventar historias. Barcelona: Hogar del Libro.

SÁNCHEZ-ENCISO, J., RINCÓN, F. (1988). Los talleres literarios. Una alternativa al historicismo. Barcelona: Montesinos.

SAVAGEAU, A.E. (2013). "Let's get personal: making sustainability tangible to students". En International Journal of Sustainability in Higher Education, 14 (1), 15-24.

ULL, À., et al. (2009). "La formación de competencias básicas para el desarrollo sostenible: el papel de la Universidad". En Revista de Educación, 1 (extra), 219237.

. (2010). "Conocimientos y actitudes del profesorado universitario sobre problemas ambientales". En Enseñanza de las Ciencias, 28 (3), 433-446.

. (2014). "Preconcepciones y actitudes del profesorado de Magisterio ante la incorporación en su docencia de competencias para la sostenibilidad". En Enseñanza de las Ciencias, 32 (2), 91112.

VILCHES PEÑA, A., GIL PÉREZ, D. (2010). “¿Cómo puede contribuir la educación a la construcción de un futuro sostenible?". En Revista Eureka sobre enseñanza y divulgación de las ciencias, 7 (extra 4), 297-315.

. (2015). "Ciencia de la Sostenibilidad: ¿Una nueva disciplina o un nuevo enfoque para todas las disciplinas?". En Revista Iberoamericana de Educación, 69 (1), 39-60. 